\title{
Terapêutica Sequencial Vs. Tripla Standard na Erradicação do Helicobacter Pylori - Qual a Evidência?
}

Rute Salomé Guedes da Mota Pereira*, Daniela Filipa de Andrade Maia**, Marisa Loio Rainho Rodrigues ${ }^{* * *}$

\section{Resumo}

Introdução: 0 tratamento clássico de erradicação do Helicobacter pylori (HP) é a terapêutica tripla standard com um inibidor da bomba de protões (IBP), claritromicina e amoxicilina ou metronidazol. Na última década verificou-se diminuição da eficácia deste esquema. Para colmatar esta perda de eficácia, tem-se assistido a um crescente interesse pela terapêutica sequencial (IBP e amoxicilina cinco dias, seguido de IBP, claritromicina e metronidazol durante cinco dias), nomeadamente em regiões com elevada resistência à claritromicina, incluindo Portugal.

Objetivo: Rever a evidência da eficácia da terapêutica sequencial de erradicação do HP comparativamente com a terapêutica standard durante dez e catorze dias.

Métodos: Pesquisa de normas de orientação clínica (NOC), revisões sistemáticas (RS), meta-análises (MA) e ensaios controlados e aleatorizados (ECA) nas principais bases de dados científicas. Utilizaram-se os termos MeSH helicobacter pylori e disease eradication, e selecionaram-se artigos publicados entre janeiro de 2009 e março de 2014, nas línguas portuguesa, inglesa e espanhola. Para avaliação dos níveis de evidência (NE) utilizou-se a escala de evidência Oxford 2011.

Resultados: Foram selecionados 8 NOC, 1 RS e MA e 2 ECA. A terapêutica sequencial é recomendada como opção no tratamento de primeira linha pela European Helicobacter Study Group e pela German Society for Digestive and Metabolic Diseases. As restantes NOC recomendam a terapêutica standard.

\begin{abstract}
Introduction: The classical treatment of Helicobacter pylori (HP) infection is standard triple therapy with a proton-pump inhibitor (PPI), clarithromycin and amoxicillin or metronidazole. In the last decade there has been a decrease in the efficacy of this regimen. In order to address this loss of efficacy, it has witnessed a growing interest in sequential therapy (PPI and amoxicillin for five days, followed by PPI, clarithromycin and metronidazole during five days), particularly in regions with high resistance to clarithromycin, including Portugal.
\end{abstract}

Objective: To review the evidence on the efficacy of sequential therapy in HP eradication compared to the standard triple therapy during ten and fourteen days.

Methods: Clinical guidelines (CG), systematic reviews $(\mathrm{SR})$, meta-analyzes (MA) and randomized controlled studies (RCS) were researched in the main scientific databases, using the MeSH terms helicobacter pylori and disease eradication. Articles published between January 2009 and March 2014 on Portuguese, Spanish and English languages were selected. Oxford 2011 evidence scale was used to evaluate the evidence levels (EL).

Results: 8 CG, 1 SR and MA and 2 RCS were selected. Sequential therapy is recommended as an option in first line treatment by European Helicobacter Study Group and German Society for Digestive and Metabolic Diseases. The remaining CG recommend standard triple therapy. SR demonstrates that sequential therapy is slightly superior to standard therapy for ten

\footnotetext{
* Interna de formação Específica em MGF na USF Santa Clara.

** Interna de formação Específica em MGF na USF Santa Clara.

*** Interna de formação Específica em MGF na USF Santa Clara.
} 
A RS demonstra que a terapêutica sequencial é ligeiramente superior à terapêutica standard de dez dias e não superior à terapêutica standard de catorze dias (NE 2). Dos ECA analisados, um sugere que a terapêutica sequencial é ligeiramente superior à terapêutica standard de catorze dias (NE 2) e outro que a terapêutica sequencial não é superior à terapêutica standard de dez dias (NE 2).

Conclusões: A terapêutica sequencial parece ser ligeiramente superior à terapêutica standard de dez dias, sem diferenças consistentes relativamente à terapêutica standard de catorze dias.

Palavras-chave: Helicobacter pylori; erradicação de doença, agentes antibacterianos.

\section{Introdução}

A infeção por Helicobacter pylori (HP) é uma das infeções crónicas mais comuns, afetando cerca de metade da população mundial., ${ }^{1,2}$ A prevalência da infeção por HP varia consoante a área geográfica, a idade, a raça, a etnia e o nível socioeconómico dos indivíduos. ${ }^{3}$ Nos países em desenvolvimento a taxa de infeção pode atingir os $70 \%$, enquanto nos países desenvolvidos é, tipicamente, inferior a $40 \%{ }^{3}$ Em Portugal estima-se que a taxa de infeção por HP seja superior a $80 \%$ na população adulta. ${ }^{4}$

A infeção por HP está quase sempre associada a inflamação da mucosa gástrica que, quando não tratada, pode evoluir para gastrite atrófica, metaplasia intestinal e displasia. A gastrite crónica associada a HP é habitualmente assintomática e o seu principal significado clínico reside na correlação estreita com úlcera péptica, carcinoma gástrico e linfoma de MALT gástrico.

O esquema de tratamento mais utilizado universalmente e preconizado pela Direção-Geral da Saúde (DGS) é a terapêutica tripla standard, constituída por um inibidor da bomba de protões (IBP), claritromicina e amoxicilina ou metronidazol. ${ }^{5}$ Apesar desta associação ser amplamente utilizada, têm surgido, na última década, estudos que revelam diminuição da sua eficácia, com taxas de erradicação inferiores a 70-80\% em alguns países. ${ }^{6} 0$ aumento da resistência à claritromicina tem sido o fator mais implicado na perda de eficácia terapêutica deste esquema. A taxa global de resistência à claritromicina na Europa aumentou de 9\% em 1998 para $17,6 \%$ em $2008-2009 .^{7}$ days and not superior to standard therapy during fourteen days (EL 2). From RCS analyzed, one suggests that sequential therapy is slightly superior to standard therapy for fourteen days (EL 2) and other shows that sequential therapy is not superior to standard therapy for ten days (EL 2).

Conclusions: Sequential therapy seems to be slightly superior to standard therapy during ten days, with no consistent differences relatively to standard therapy during fourteen days.

Keywords: Helicobacter pylori; disease eradication, anti-bacterial agents.

No sentido de colmatar a perda de eficácia terapêutica do esquema standard tem-se assistido a um crescente interesse por outras terapêuticas de erradicação, nomeadamente pela terapêutica sequencial, que consiste em cinco dias de tratamento com IBP e um antibiótico (geralmente amoxicilina), seguidos de cinco dias com IBP associado a dois antibióticos (geralmente claritromicina e um nitroimidazol). Esta terapêutica tem sido cada vez mais usada, principalmente nas regiões com maior taxa de resistência à claritromicina, como é o caso de Portugal. ${ }^{8}$

\section{Objetivo}

Rever a evidência disponível quanto à eficácia da terapêutica sequencial de erradicação do HP comparativamente com a terapêutica tripla standard durante dez e catorze dias.

\section{Métodos}

Foram pesquisadas normas de orientação clínica (NOC), revisões sistemáticas (RS), meta-análises (MA) e ensaios controlados e aleatorizados (ECA) nas bases de dados MEDLINE, National Guideline Clearinghouse, Guidelines Finder, Canadian Medical Association Practice, Guidelines InfoBase, The Cochrane Library, Clinical Evidence, $D A R E$, no sitio da DGS e referências bibliográficas dos artigos selecionados. Foram selecionados artigos publicados entre 1 de janeiro de 2009 e 31 de março de 
2014, nas línguas portuguesa, inglesa e espanhola. Utilizaram-se os termos MeSH helicobacter pylori e disease eradication. Para avaliação dos níveis de evidência (NE) foi utilizada a escala de Oxford $2011 .^{9}$

Incluíram-se os estudos em que o diagnóstico da infeção por HP e a confirmação da erradicação fossem realizados com pelo menos um dos seguintes exames: teste respiratório da ureia, teste do antigénio fecal, histologia, teste rápido da urease ou cultura. Foram excluídos os estudos que comparassem a terapêutica sequencial com a terapêutica tripla standard durante sete dias e com outras terapêuticas, que incluíssem pessoas com comorbilidades significativas (insuficiência renal, insuficiência hepática e cancro), casos de recidiva, pessoas com idade inferior a dezoito anos e estudos in vitro e em animais.

\section{Resultados}

Após realização da pesquisa foram encontrados 373 artigos, tendo sido excluídos 362 de acordo com os critérios de exclusão, por estarem repetidos ou por estarem incluídos na RS e MA. Foram selecionados para esta revisão 11 artigos: 8 NOCs, 1 RS e MA e 2 ECA (Quadro 1, 2 e 3).

\section{Normas de orientação clínica (Quadro 1)}

A maioria das NOC incluídas nesta revisão não recomenda a terapêutica sequencial como tratamento de primeira linha na erradicação do HP.

O Toward Optimized Practice Program (2009) ${ }^{10}$, a Asia-Pacific Association of Gastroenterology (2009) ${ }^{11}$, a Japanese Society for Helicobacter Research $(2010)^{12}$ e a Danish Society for Gastroenterology (2011) ${ }^{13}$ recomendam a terapêutica tripla standard durante sete dias como tratamento de primeira linha. A World Gastroenterology Organisation (2010) ${ }^{14}$ emitiu uma NOC para os países em desenvolvimento que recomenda a terapêutica tripla durante sete, dez ou catorze dias. A Direção-Geral da Saúde $(2011)^{5}$ recomenda a terapêutica tripla standard durante dez a catorze dias.

Por outro lado, a German Society for Digestive and Metabolic Diseases (2009) ${ }^{15}$ e a European Helicobacter Study Group (2012) ${ }^{7}$ consideram a terapêutica sequencial como uma alternativa no tratamento de primeira linha na erradicação do HP. Segundo a German Society for Digestive and Metabolic Diseases, o tratamento de primeira linha na erradicação do HP deve consistir na terapêutica tripla durante pelo menos sete dias. Em alternativa poderá ser utilizada a terapêutica sequencial ou a terapêutica quádrupla concomitante. A NOC do European Helicobacter Study Group, o designado Consenso de Maastricht IV, é a primeira a recomendar esquemas terapêuticos diferentes consoante a taxa de resistência à claritromicina para cada região. Assim, para as regiões de baixa resistência à claritromicina (inferior a 15\%) é recomendada a terapêutica tripla standard ou, em alternativa, a terapêutica quádrupla com bismuto. Para as regiões de elevada resistência à claritromicina (>15\%) o tratamento de primeira linha deverá consistir na terapêutica quádrupla com bismuto. No caso de não estarem disponíveis compostos com bismuto nestas regiões, como ocorre em Portugal, é recomendada a terapêutica sequencial ou terapêutica quadrupla concomitante.

De notar, que apenas as NOC da Asia-Pacific Association of Gastroenterology e da German Society for Digestive and Metabolic Diseases são baseadas em evidência. 
Quadro 1: Normas de Orientação Clínica relativas a tratamento de $1^{\text {a }}$ linha para erradicação do HP.

\begin{tabular}{|c|c|c|}
\hline NOC & Ano & Recomendação \\
\hline $\begin{array}{l}\text { Toward Optimized Practice Program } \\
\text { (Canadá) } \\
\text { Consenso de Peritos }\end{array}$ & 2009 & Terapêutica tripla, 7 dias. \\
\hline $\begin{array}{l}\text { Asia-Pacific Association of } \\
\text { Gastroenterology (Ásia) } \\
\text { Baseada em evidência }\end{array}$ & 2009 & $\begin{array}{l}\text { Terapêutica tripla, pelo menos } 7 \text { dias. } \\
\text { Não existem dados suficientes para recomendar a } \\
\text { terapêutica sequencial como tratamento de primeira linha. }\end{array}$ \\
\hline $\begin{array}{l}\text { Japanese Society for Helicobacter } \\
\text { Research (Japão) } \\
\text { Consenso de peritos }\end{array}$ & 2010 & $\begin{array}{l}\text { Terapêutica tripla, } 7 \text { dias. } \\
\text { Não existem dados suficientes para recomendar a } \\
\text { terapêutica sequencial como tratamento de primeira linha. }\end{array}$ \\
\hline $\begin{array}{l}\text { Danish Society for Gastroenterology } \\
\text { (Dinamarca) } \\
\text { Consenso de peritos }\end{array}$ & 2011 & $\begin{array}{l}\text { Terapêutica tripla, } 7 \text { dias. } \\
\text { Terapêutica sequencial pode ser uma alternativa no caso } \\
\text { de falência terapêutica. }\end{array}$ \\
\hline $\begin{array}{l}\text { World Gastroenterology Organisation } \\
\text { (países em desenvolvimento) } \\
\text { Consenso de peritos }\end{array}$ & 2010 & $\begin{array}{l}\text { Terapêutica tripla, } 7,10 \text { ou } 14 \text { dias. } \\
\text { Alternativa: Terapêutica quádrupla com bismuto. }\end{array}$ \\
\hline $\begin{array}{l}\text { Direção-Geral da Saúde } \\
\text { (Portugal) } \\
\text { Consenso de peritos }\end{array}$ & 2011 & Terapêutica tripla, 10 a 14 dias. \\
\hline $\begin{array}{l}\text { German Society for Digestive and } \\
\text { Metabolic Diseases (Alemanha) } \\
\text { Baseada em evidência }\end{array}$ & 2009 & $\begin{array}{l}\text { Terapêutica tripla, pelo menos } 7 \text { dias. } \\
\text { Alternativa: terapêutica sequencial ou terapêutica } \\
\text { quádrupla concomitante, } 7 \text { dias. }\end{array}$ \\
\hline $\begin{array}{l}\text { European Helicobacter Study Group } \\
\text { (Maastricht IV) } \\
\text { (Europa) } \\
\text { Consenso de peritos }\end{array}$ & 2012 & $\begin{array}{l}\text { Regiões de baixa resistência à claritromicina: terapêutica } \\
\text { tripla ou terapêutica quádrupla com bismuto (alternativa). } \\
\text { Regiões de elevada resistência à claritromicina: } \\
\text { terapêutica quádrupla com bismuto ou terapêutica } \\
\text { sequencial (alternativa) ou terapêutica quádrupla } \\
\text { concomitante (alternativa). }\end{array}$ \\
\hline
\end{tabular}

\section{Revisão sistemática e meta-análise (Quadro 2)}

A meta-análise de Gatta L, et al. $(2013)^{16}$ engloba 21 estudos, sendo que catorze ECA comparam a terapêutica sequencial com a terapêutica tripla standard de dez dias e sete ECA comparam a terapêutica sequencial com a terapêutica tripla standard de catorze dias. A terapêutica sequencial mostrou ser ligeiramente superior à terapêutica tripla standard de dez dias, mas não superior à de catorze dias. Os efeitos secundários foram comparáveis entre os grupos. É de notar a heterogeneidade dos estudos incluídos, que diferem no país de origem, no tipo de IBP utilizado e no uso de tinidazol ou metronidazol. Desta forma, atribui-se um NE 2. 
Quadro 2: Revisão sistemática e meta-análise.

\begin{tabular}{|c|c|c|c|c|}
\hline Referência & Estudos & Intervenção & Resultados & NE \\
\hline \multirow{2}{*}{$\begin{array}{l}\text { Gatta L, et al. } \\
2013\end{array}$} & $\begin{array}{l}14 \text { ECA } \\
(n=2746)\end{array}$ & $\begin{array}{l}\text { Seq. } 10 \text { dias } \\
(n=1368) \\
\text { Vs. } \\
\text { Tripla standard } \\
10 \text { dias } \\
(n=1378)\end{array}$ & $\begin{array}{l}\text { Terapêutica seq. é ligeiramente superior } \\
\text { à terapêutica tripla standard de } 10 \text { dias } \\
\text { (resultados heterogéneos): } \\
\text { Taxa erradicação seq. } 84,3 \% \text { (IC } 95 \%=79,8 \\
\text { a } 88,4 \text { ) vs. standard } 75,3 \% \text { (IC } 95 \%=69,6 \text { a } \\
77,9)(R R=1,11 ; \text { IC } 95 \%=1,04 \text { a 1,19) } \\
\text { Efeitos secundários comparáveis (11 ECA): } \\
\text { RR=0,94 (IC } 95 \%=0,79 \text { a 1,13) }\end{array}$ & \\
\hline & $\begin{array}{l}7 \text { ECA } \\
(n=2451)\end{array}$ & $\begin{array}{l}\text { Seq. } 10 \text { dias } \\
(n=1224) \\
\text { Vs. } \\
\text { Tripla standard } \\
14 \text { dias } \\
(n=1227)\end{array}$ & $\begin{array}{l}\text { Terapêutica seq. não é superior à terapêutica } \\
\text { tripla standard de } 14 \text { dias } \\
\text { (resultados heterogéneos): } \\
\text { Taxa erradicação sequencial } 80,8 \% \text { (IC } 95 \%=76 \\
\text { a 85,1) vs. standard } 81,3 \% \text { (IC } 95 \%=79,5 \text { a } \\
84,7) \text { (RR=1,00; IC } 95 \%=0,94 \text { a 1,06) } \\
\text { Efeitos secundários comparáveis (4 ECA): } \\
\text { RR=0,98 (IC } 95 \%=0,73 \text { a 1,33) }\end{array}$ & \\
\hline
\end{tabular}

Legenda: IC - intervalo de confiança; ECA - ensaio controlado e aleatorizado; NE - nível de evidência; RR - risco relativo; seq. - sequencial; vs. - versus.

\section{Ensaios controlados aleatorizados (Quadro 3)}

Nasa M, et al. (2013) $)^{17}$ compararam a terapêutica sequencial com a terapêutica tripla standard de catorze dias, tendo sido constatado que a terapêutica sequencial foi ligeiramente superior à tripla standard. A este ECA foi atribuído um NE 2.
Zhou L, et al. (2014) ${ }^{18}$ compararam a terapêutica sequencial com a terapêutica tripla standard de 10 dias, sendo que terapêutica sequencial não se mostrou superior. A este ECA foi também atribuído um NE 2.

Os efeitos secundários foram comparáveis em ambos os estudos. 
Quadro 3: Ensaios controlados e aleatorizados.

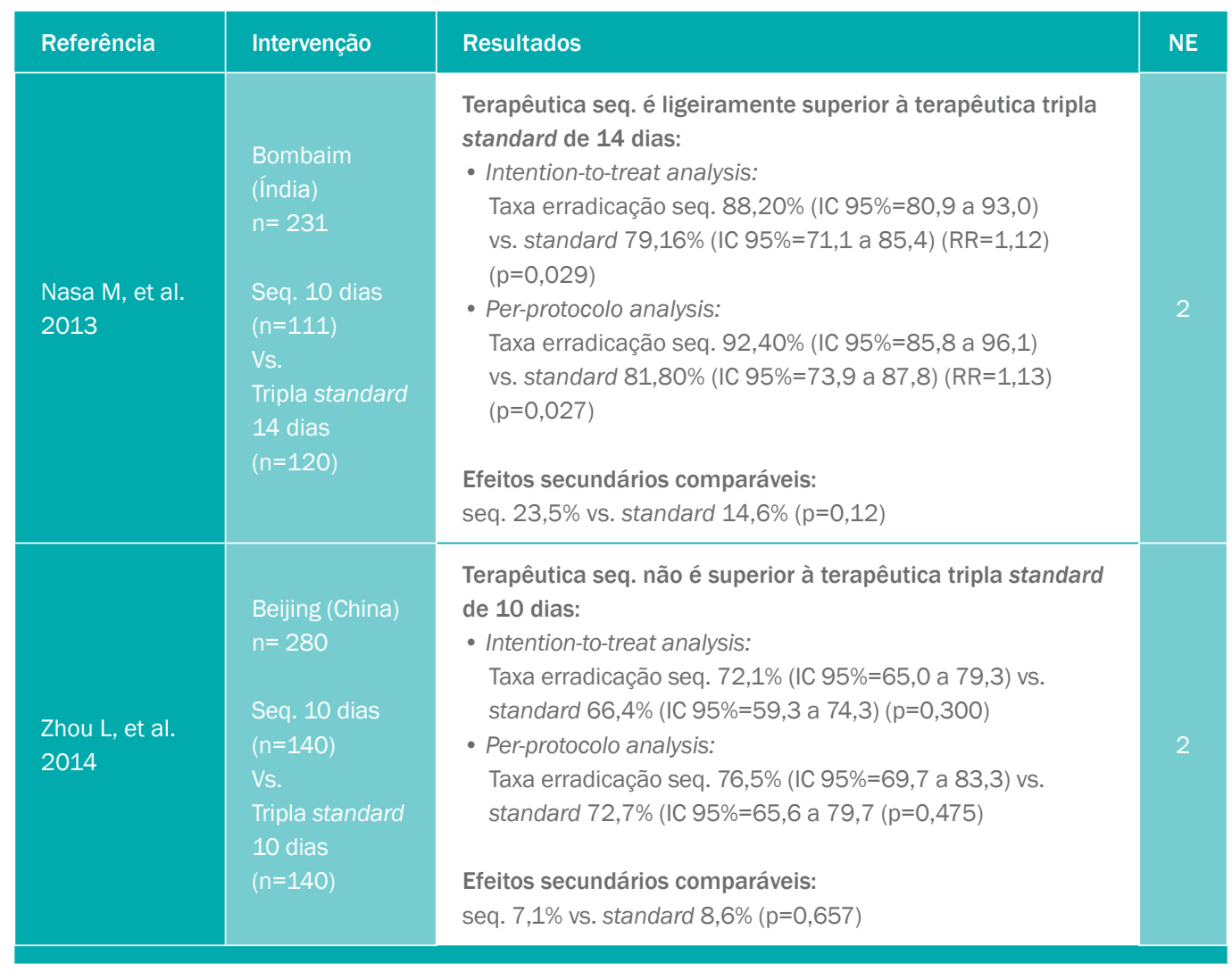

Legenda: IC - intervalo de confiança; NE - nível de evidência; $p$ - p value; RR - risco relativo; seq. - sequencial. vs. - versus.

\section{Conclusões}

A terapêutica de erradicação do HP recomendada em Portugal consiste na terapêutica tripla standard. ${ }^{5}$ No entanto, este esquema tem mostrado taxas de erradicação sucessivamente mais baixas, o que se deve sobretudo ao aumento da prevalência de estirpes resistentes à claritromicina. A terapêutica sequencial tem sido cada vez mais utilizada, de forma a obter maiores taxas de erradicação. Teoricamente este esquema será mais eficaz porque a amoxicilina numa fase inicial do tratamento permite enfraquecer a parede celular bacteriana, o que impede o desenvolvimento de canais de efluxo de fármacos como a claritromicina, aumentando a eficácia da mesma na segunda fase do tratamento. ${ }^{19}$

Esta revisão conclui que a terapêutica sequencial parece ser ligeiramente superior à terapêutica tripla standard de dez dias, não tendo sido demonstradas diferenças consistentes relativamente à terapêutica tripla standard de catorze dias. De salientar a grande heterogeneidade dos resultados obtidos nos estudos, uma vez que estes foram desenvolvidos em diferentes regiões do mundo, com taxas de resistência aos antibióticos variáveis.
Existem possíveis desvantagens associadas à terapêutica sequencial, uma vez que se trata de um esquema mais complexo, hipoteticamente com maior possibilidade de toma incorreta e de menor adesão terapêutica. ${ }^{20}$ Além disso, em caso de falência do tratamento, as opções terapêuticas ficam mais limitadas, uma vez que já foram utilizados três antibióticos. ${ }^{21}$

A escolha do regime terapêutico deve basear-se no conhecimento da prevalência de estirpes de HP resistentes na comunidade e nos antecedentes pessoais do doente. ${ }^{6}$ Desta forma, são necessários mais estudos, com maior homogeneidade, que avaliem a eficácia da terapêutica sequencial na Europa Ocidental, nomeadamente em Portugal. Além disso, é fundamental a investigação de novos esquemas terapêuticos uma vez que é previsível uma perda progressiva de eficácia dos esquemas disponíveis com o aumento de resistência à claritromicina e aos nitroimidazois. 


\section{Referências Bibliográficas}

1. Cave DR. Transmission and epidemiology of Helicobacter pylori. Am J Med 1996; 100:12-17.

2. Cave DR. How is Helicobacter pylori transmitted? Gastroenterology 1997; 113(6):9-14.

3. Brown LM. Helicobacter pylori: epidemiology and routes of transmission. Epidemiol Rev 2000; 22: 283-297.

4. Azevedo NF, Guimarães N, Figueiredo C, Keevil CW, Vieira MJ. A New Model for the Transmission of Helicobacter pylori: Role of Environmental Reservoirs as Gene Pools to Increase Strain Diversity. Crit Rev Microbiol 2007; 33(3):157-69.

5. Direção-Geral da Saúde. Supressão Ácida: Utilização dos Inibidores da Bomba de Protões e das suas Alternativas Terapêuticas. Norma número 036/2011. Lisboa: Direção-Geral da Saúde; 2011 Setembro 30.

6. Georgopoulos S, Papastergiou V, Karatapanis S. Helicobacter pylori Eradication Therapies in the Era of Increasing Antibiotic Resistance: A Paradigm Shift to Improved Efficacy. Gastroenterol Res Pract 2012.

7. Malfertheiner P, Megraud F, O'Morain CA, et al. Management of Helicobacter pylori infection - the Maastricht IV/ Florence Consensus Report. The European Helicobacter Study Group. Gut 2012; 61:646-664.

8. Oleastro M, Furtado C, Andrea Santos A, João Benoliel, Ratilal P, Liberato M. Resistência primária de Helicobacter pylori em doentes sintomáticos de dois hospitais da região de Lisboa. Boletim epidemiológico, Instituto Nacional de Saúde Dr Ricardo Jorge. 2014.

9. Centre for Evidence-based Medicine [Internet]. Oxford Centre for Evidence-based Medicine Levels of Evidence. 2011. Disponível em: http://www.cebm.net/ocebm-levels-of-evidence.

10. Toward Optimized Practice Program. Guideline for the Treatment of Helicobacter Pylori Infection in Adults. Alberta: Toward Optimized Practice Program; 2009.

11. Fock KM, Katelaris P, Sugano K, et al. Second Asia-Pacific Consensus Guidelines for Helicobacter pylori infection.
Asia-Pacific Association of Gastroenterology. Journal of Gastroenterology and Hepatology 2009; 24: 1587-1600.

12. Asaka M, Kato M, Takahashi S, et al. Guidelines for the Management of Helicobacter pylori Infection in Japan: 2009 Revised Edition. Japanese Society for Helicobacter Research. Helicobacter 2010; 15: 1-20.

13. Bytzer P, Dahlerup JF, Eriksen JR, et al. Diagnosis and treatment of Helicobacter pylori infection. Danish Society for Gastroenterology. Dan Med Bull 2011; 58(4): 4271.

14. World Gastroenterology Organisation. Helicobacter pylori nos países em desenvolvimento. World Gastroenterology Organisation Practice Guidelines; 2010.

15. Fischbach W, Malfertheiner P, Hoffmann JC, et al. Helicobacter Pylori and Gastroduodenal Ulcer Disease. German Society for Digestive and Metabolic Diseases. Dtsch Arztebl Int 2009; 106(49): 801-808.

16. Gatta L, Vakil N, Vaira D, Scarpignato C. Global eradication rates for Helicobacter pylori infection: systematic review and meta-analysis of sequential therapy. BMJ 2013 Aug; 347.

17. Nasa M, Choksey A, Phadke A, Sawant P. Sequential therapy versus standard triple-drug therapy for Helicobacter pylori eradication: A randomized trial. Indian J Gastroenterol. 2013 Nov;32(6):392-6.

18. Zhou L, Zhang J, Chen M, et al. A Comparative Study of Sequential therapy and standard triple-drug therapy for Helicobacter pylori infection: A Randomized Multicenter Trial. Am J Gastroenterol. 2014 Apr;109(4):535-41.

19. Federico A, Gravina AG, Miranda A, Loguercio C, Romano M. Eradication of infection: Which regimen first? World journal of gastroenterology : WJG. 2014;20(3):665-72.

20. Kanizaj TF, Kunac N. Helicobacter pylori : Future perspectives in therapy reflecting three decades of experience. World J Gastroenterol 2014 Jan 21; 20(3): 699-705.

21. Gisbert JP. Rescue Therapy for Helicobacter pylori Infection 2012. Gastroenterology Research and Practice 2012.

\section{Conflito de Interesses}

As autoras declaram não ter conflitos de interesses.

\section{Endereço para Correspondência:}

Rute Salomé Guedes da Mota Pereira

Email: rutemota7@gmail.com

Rua Manuel José Santos Leite, $n^{\circ} 2616^{\circ}$ Dto

4470-795 Maia 